\title{
Locally primitive normal Cayley graphs of metacyclic groups
}

\author{
Jiangmin Pan* \\ Department of Mathematics, School of Mathematics and Statistics, \\ Yunnan University, Kunming 650031, P. R. China \\ jmpan@ynu.edu.cn \\ Submitted: Mar 3, 2008; Accepted: Jul 28, 2009; Published: Aug 7, 2009 \\ Mathematics Subject Classifications: 05C25.
}

\begin{abstract}
A complete characterization of locally primitive normal Cayley graphs of metacyclic groups is given. Namely, let $\Gamma=\operatorname{Cay}(G, S)$ be such a graph, where $G \cong \mathbb{Z}_{m} \cdot \mathbb{Z}_{n}$ is a metacyclic group and $m=p_{1}^{r_{1}} p_{2}^{r_{2}} \cdots p_{t}^{r_{t}}$ such that $p_{1}<p_{2}<\cdots<p_{t}$. It is proved that $G \cong D_{2 m}$ is a dihedral group, and $\operatorname{val}(\Gamma)=p$ is a prime such that $p \mid\left(p_{1}\left(p_{1}-1\right), p_{2}-1, \ldots, p_{t}-1\right)$. Moreover, three types of graphs are constructed which exactly form the class of locally primitive normal Cayley graphs of metacyclic groups.
\end{abstract}

\section{Introduction}

Throughout the paper, groups are finite, and graphs are finite, simple and undirected. For a graph $\Gamma$, let $V(\Gamma)$ denote its vertex set. For $v \in V(\Gamma)$, let $\Gamma(v)$ denote the set of vertices which are adjacent to $v$. If $\Gamma$ is regular, then $|\Gamma(v)|$ is called the valency of $\Gamma$, and denoted by $\operatorname{val}(\Gamma)$. A digraph $\Gamma$ is called a Cayley digraph if there exist a group $G$ and a subset $S \subseteq G \backslash\{1\}$, such that its vertex set can be identified with $G$, and two vertices $u, v$ are adjacent if and only if $v u^{-1} \in S$. If further $S=S^{-1}=:\left\{s^{-1} \mid s \in S\right\}$, then $\Gamma$ is undirected and called Cayley graph. This Cayley digraph is denoted by Cay $(G, S)$, and the vertex of $\Gamma$ corresponding to the identity element of group $G$ is denoted by $\mathbf{1}$.

Let $\Gamma$ be a graph, and let $X$ be a group of automorphisms of $\Gamma$, that is, $X 6$ Aut $\Gamma$. Then $\Gamma$ is called $X$-vertex transitive (or simply called vertex transitive) if $X$ is transitive on $V(\Gamma)$, and $\Gamma$ is called $X$-locally primitive (or simply called locally primitive) if $X_{v}$ : = $\left\{x \in X \mid v^{x}=v\right\}$ is primitive on $\Gamma(v)$ for each vertex $v$. A 2-arc of $\Gamma$ is a sequence $(u, v, w)$

*This work was partially supported by NNSF(K1020261) and YNSF(2008CD060). 
of three distinct vertices such that $v$ is adjacent to both $u$ and $w$. Then $\Gamma$ is called $(X, 2)$ arc-transitive, if $X$ is transitive on the set of 2-arcs of $\Gamma$. It is known that 2-arc-transitive graphs form a proper subclass of vertex transitive locally primitive graphs.

It is well-known that a graph $\Gamma$ is a Cayley graph of a group $G$ if and only if Aut $\Gamma$ contains a subgroup which is isomorphic to $G$ and regular on $V(\Gamma)$, see [3, Proposition 16.3]. If Aut $\Gamma$ has a normal subgroup which is regular and isomorphic to $G$, then $\Gamma$ is called a normal Cayley graph of $G$.

Locally primitive Cayley graphs and 2-arc-transitive Cayley graphs have been extensively studied, see for example, $[1,7,9,11,12]$ and references therein. Also, normal Cayley graphs have received much attention in the literature, see for example, [6, 8, 13]. In particular, 2-arc-transitive normal Cayley graphs of elementary abelian groups are classified by Ivanov and Praeger [6]. This motivates the author to study locally primitive normal Cayley graphs of some classes of groups. The purpose of this paper is to give a complete characterization of locally primitive normal Cayley graphs of metacyclic groups. For convenience, we limit our attention on nonabelian metacyclic group case as abelian metacyclic group case is trivial.

We now state the main theorems of this paper.

Theorem 1.1 Let $\Gamma=\operatorname{Cay}(G, S)$ be a connected $X$-locally primitive normal Cayley graph of valency at least 3 , where $G \cong \mathbb{Z}_{m} \cdot \mathbb{Z}_{n}$ is a nonabelian metacyclic group, and $\hat{G}<X 6$ Aut $(\Gamma)$ with $\hat{G}=\{\hat{g}: x \rightarrow x g$ for all $x \in G \mid g \in G\}$. Let $m=p_{1}^{r_{1}} p_{2}^{r_{2}} \cdots p_{t}^{r_{t}}$ be the standard decomposition of $m$ such that $p_{1}<p_{2}<\cdots<p_{t}$. Then $G \cong D_{2 m}$ is a dihedral group, and $\operatorname{val}(\Gamma)=p$ is a prime such that $p \mid\left(p_{1}\left(p_{1}-1\right), p_{2}-1, \ldots, p_{t}-1\right)$. Further, either $m$ is a prime and $\mathbb{Z}_{p} 6 X_{1} 6 \mathbb{Z}_{p}: \mathbb{Z}_{p-1}$, or $X_{1}=\mathbb{Z}_{p}$.

Theorem 1.1 has the following interesting corollary which shows 2-arc-transitive normal Cayley graphs of metacyclic groups are complete bi-partite graphs of prime valency.

Corollary 1.2 Every 2-arc-transitive normal Cayley graph of a metacyclic group is isomorphic to $\mathrm{K}_{p, p}$, where $p$ is a prime.

In section 4, we will construct three types of Cayley graphs (see Constructions 4.1-4.3 below). The second main theorem of this paper is to prove that all locally primitive normal Cayley graphs of metacyclic groups are in these constructions.

Theorem 1.3 Graphs in Constructions 4.1 - 4.3 are exactly form the class of locally primitive normal Cayley graphs of metacyclic groups.

This paper is organized as follows. Section 2 gives some necessary preliminaries and lemmas. Section 3 proves Theorem 1.1. Section 4 first constructs three types of graphs, and then proves Theorem 1.3. Section 5 gives a characterization of locally primitive bi-normal Cayley graphs of dihedral groups. 


\section{Preliminaries}

In this section, we give some necessary preliminaries and lemmas for proving Theorem 1.1 and Theorem 1.3.

Let $\Gamma=\operatorname{Cay}(G, S)$. Let

$$
\begin{gathered}
\hat{G}=\{\hat{g}: x \rightarrow x g \text { for all } x \in G \mid g \in G\}, \\
\text { Aut }(G, S)=\left\langle\sigma \in \operatorname{Aut}(G) \mid S^{\sigma}=S\right\rangle .
\end{gathered}
$$

It is known that $\hat{G}$ and $\operatorname{Aut}(G, S)$ are subgroups of $\operatorname{Aut}(\Gamma)$, and $\operatorname{Aut}(G, S)$ fixes the vertex $\mathbf{1}$ and normalizes the regular subgroup $\hat{G}$. Moreover, the normalizer of $\hat{G}$ in $\operatorname{Aut}(\Gamma)$ is uniquely determined by $\operatorname{Aut}(G, S)$.

Lemma 2.1 ([5, Lemma 2.1]) For a Cayley graph $\Gamma=\operatorname{Cay}(G, S)$, the normalizer $\mathbf{N}_{\text {Aut }(\Gamma)}(\hat{G})=\hat{G}$ :Aut $(G, S)$. In particular, if $\Gamma$ is a normal Cayley graph of group $G$, then $\operatorname{Aut}(\Gamma)=\hat{G}: \operatorname{Aut}(G, S)$.

For a given graph, it is generally quite difficult to describe its automorphism group. However, for a Cayley graph $\Gamma=\operatorname{Cay}(G, S)$, by Lemma 2.1, the automorphism group Aut $(\Gamma)$ has a transitive subgroup $\mathbf{N}_{\text {Aut }(\Gamma)}(\hat{G})$ which contains a regular subgroup $\hat{G}$ and can be described in terms of Aut $(G, S)$. Thus the subgroup $\operatorname{Aut}(G, S)$ plays an important role in the study of Cayley graphs, see for example, $[5,10,13,15]$. If $\Gamma=\operatorname{Cay}(G, S)$ is an $X$-normal Cayley graph, then by Lemma 2.1, the vertex stabilizer $X_{1} 6 \operatorname{Aut}(G, S)$, that is, $X_{1}$ acts on $\Gamma$ in a very nice way-by conjugation. In particular, if $\Gamma$ is connected, the action of $X_{\mathbf{1}}$ on $\Gamma$ is uniquely determined by its action on $\Gamma(\mathbf{1})=S$. Due to this nice action, the following lemma is not difficult to prove.

Lemma 2.2 Let $\Gamma=\operatorname{Cay}(G, S)$ be a connected $X$-locally primitive normal Cayley graph, where $\hat{G}<X 6$ Aut $(\Gamma)$. Then

(1) $\langle S\rangle=G$, and all elements of $S$ are involutions and conjugate under Aut $(G, S)$.

(2) $X_{1} 6 \operatorname{Aut}(G, S)$ acts faithfully and primitively on $S$.

By Lemma 2.2, we have following method for constructing arc-transitive normal Cayley graphs.

Construction. For a group $G$, take an element $g \in G \backslash\{1\}$ and a subgroup $H$ of Aut $(G)$. Let $S=\left\{g^{h} \mid h \in H\right\}$ and $\Gamma=\operatorname{Cay}(G, S)$.

Lemma 2.3 Using notations as in the above construction. Then $\Gamma$ is an arc-transitive normal Cayley digraph of $G$. In particular, if $\langle S\rangle=G$ and $g$ is conjugate to $g^{-1}$ in $H$, then $\Gamma$ is a connected undirected arc-transitive normal Cayley graph of $G$.

Proof. Let $X=\hat{G}$ :Aut $(G, S)$. Then $\hat{G} \triangleleft X$ and $X_{1}=\operatorname{Aut}(G, S) \supseteq H$, so $X_{1}$ is transitive on $\Gamma(\mathbf{1})=S$, thus $\Gamma$ is an $X$-arc-transitive normal Cayley digraph of $G$. The last statement is then obviously true. 


\section{Proof of Theorem 1.1}

We first prove two technical lemmas for proving Theorem 1.1. The first plays an important role which reduces the metacyclic group case into dihedral group case.

Lemma 3.1 Let $\Gamma=\operatorname{Cay}(G, S)$ be a connected $X$-locally primitive normal Cayley graph of valency at least 3 , where $G \cong \mathbb{Z}_{m} \cdot \mathbb{Z}_{n}$ is a nonabelian metacyclic group, and $\hat{G}<X 6$ Aut $(\Gamma)$. Then $n=2$ and $G \cong D_{2 m}$ is a dihedral group.

Proof. By Lemma 2.2, $\langle S\rangle=G$, and all elements of $S$ are involutions and conjugate under Aut $(G, S)$. Since $G \cong \mathbb{Z}_{m} \cdot \mathbb{Z}_{n}$ is a metacyclic group, we may suppose that

$$
G=\left\langle a, b \mid a^{m}=b^{n k}=1, b^{n}=a^{l}, a^{b}=a^{r}\right\rangle,
$$

where $k l=m, r^{n} \equiv 1(\bmod m)$ and $k \mid(r-1)$, see [2, P.175]. In particular, each element $x$ of $G$ can be uniquely expressed in the form $x=a^{i} b^{j}$, where $06 i 6 m-1,06 j 6 n-1$.

Suppose $x=a^{i} b^{j} \in G$ is an involution. Then $x^{2}=\left(a^{i} b^{j}\right)^{2}=a^{i}\left(b^{j} a^{i} b^{-j}\right) b^{2 j}=1$. Note $a^{i}\left(b^{j} a^{i} b^{-j}\right) \in\langle a\rangle$, it follows that $b^{2 j} \in\langle a\rangle$, and then $2 j \equiv 0(\bmod n)$. If $n$ is odd, we have $j \equiv 0(\bmod n)$, and then $x=a^{i} \in\langle a\rangle$, so $G$ has at most one involution (depending on whether $m$ is odd or even), which is impossible as $|S| ; 3$. Thus $n$ is even. Let $n=2 n_{1}$. Then $j \equiv 0\left(\bmod n_{1}\right)$, so $x=a^{i} b^{n_{1}}$ or $x=a^{m / 2}$ and $m$ is even. If $n_{1} \neq 1$, then $\langle S\rangle \subseteq\left\langle a, b^{n_{1}}\right\rangle \cong \mathbb{Z}_{m} \cdot \mathbb{Z}_{2}$ is a proper subgroup of $G$, which is again impossible as $\langle S\rangle=G$. Hence $n_{1}=1, n=2$ and $G \cong \mathbb{Z}_{m} \cdot \mathbb{Z}_{2}$ is an extension of a cyclic group by a cyclic group of order 2 .

Assume that $m$ is even. Recall that $G$ can be expressed as in (1) with $n=2$. If $r=-1$, let $a^{i} b^{-1}$ be an involution in $G \backslash\langle a\rangle$, then $\left(a^{i} b^{-1}\right)^{2}=a^{(r+1) i-l}=a^{-l}=1$, we conclude $l=m$ and $k=1$, that is, $G \cong D_{2 m}$. Thus assume now $r \neq-1$. It is easily shown that an element $a^{i} b^{-1}$ is an involution if and only if $i$ is the solution of the equation

$$
(r+1) x \equiv l(\bmod m)
$$

Since $G \backslash\langle a\rangle$ has at least two involutions, the equation (2) has solutions, say $x=i_{0}$ is one. Then all involutions of $G$ are $a^{m / 2}, a^{i_{0}} b^{-1}, \ldots, a^{i_{0}+(d-1) m / d} b^{-1}$, where $d=(r+1, m)$. Moreover, as $r \neq-1$, it follows that $d 6 \mathrm{~m} / 2$.

Since $r^{2} \equiv 1(\bmod m)$, and $m$ is even, it follows $r$ is odd, and then $4 \mid m$. For each $\sigma \in \operatorname{Aut}(G)$ and $c \in G$, it is easy to verify $\sigma\left(c^{2}\right) \in\langle a\rangle$, and then $\sigma\left(a^{m / 2}\right) \in\langle a\rangle$ as $m / 2$ is even. Note $a^{m / 2}$ is the unique involution in $\langle a\rangle$, it follows that $\sigma$ fixes $a^{m / 2}$. So $a^{m / 2} \notin S$ as $X_{1} 6$ Aut $(G, S)$ is transitive on $S$.

Now, note that $\left\langle a^{m / d}\right\rangle \triangleleft G$, we have

$$
\begin{aligned}
\langle S\rangle & \subseteq\left\langle a^{i_{0}} b^{-1}, a^{i_{0}+m / d} b^{-1}, \ldots, a^{i_{0}+(d-1) m / d} b^{-1}\right\rangle \\
& =\left\langle a^{m / d}, a^{i_{0}} b^{-1}\right\rangle=\left\langle a^{m / d}\right\rangle \cdot\left\langle a^{i_{0}} b^{-1}\right\rangle \cong \mathbb{Z}_{d} \cdot \mathbb{Z}_{2}
\end{aligned}
$$

is a proper subgroup of $G$, which is impossible as $\langle S\rangle=G$. 
Hence, $m$ is odd. Then all Sylow subgroups of $G$ are cyclic. By [14, P. 281] or [16, Theorem 6.2], we may suppose that

$$
G=\left\langle a, b \mid a^{m}=b^{2}=1, a^{b}=a^{r}\right\rangle,
$$

where $r^{2} \equiv 1(\bmod m)$ and $(2(r-1), m)=1$. Then $r \equiv-1(\bmod m)$, that is, $G \cong D_{2 m}$. This completes the proof of the lemma.

For a group $H$ and its subgroup $K$, the core of $K$ in $H$, denoted by core ${ }_{H}(K)$, is the largest normal subgroup of $H$ contained in $K . K$ is called a core-free subgroup of $H$ if core $_{H}(K)=1$. By definition, if $H$ is abelian, and has a core-free maximal subgroup $K$, then $H$ is a cyclic group of prime order and $K=1$. The following lemma gives some properties of the automorphism groups of dihedral groups.

Lemma 3.2 Let $G \cong D_{2 m}$ be a dihedral group. Let $H$ be a nontrivial subgroup of Aut $(G)$, and $K$ be a core-free maximal subgroup of $H$. Then $|H: K|$ is a prime.

Proof. Suppose that $G=\left\langle a, b \mid a^{m}=b^{2}=1, a^{b}=a^{-1}\right\rangle$. Note $\langle a\rangle$ is a characteristic subgroup of $G$, it easily follows that each automorphism $\sigma$ of $G$ has the following form:

$$
\sigma: a \rightarrow a^{i}, b \rightarrow a^{j} b \text { with }(i, m)=1 \text { and } 06 j 6 m-1 .
$$

Let

$$
\begin{gathered}
N=\left\{\sigma: a \rightarrow a, b \rightarrow a^{j} b \mid 06 j 6 m-1\right\}, \\
R=\left\{\sigma: a \rightarrow a^{i}, b \rightarrow b \mid(i, m)=1\right\} .
\end{gathered}
$$

Then it is easily shown that $N \cong \mathbb{Z}_{m} \triangleleft \operatorname{Aut}(G), R \cong \operatorname{Aut}\left(\mathbb{Z}_{m}\right)$ and $\operatorname{Aut}(G)=N: R \cong$ $\mathbb{Z}_{m}: \operatorname{Aut}\left(\mathbb{Z}_{m}\right)$.

Assume first that $H \cap N=1$. Then

$$
H=H /(H \cap N) \cong H N / N 6 \operatorname{Aut}(G) / N \cong \operatorname{Aut}\left(\mathbb{Z}_{m}\right)
$$

is abelian, so $K=1$ and $H$ is a cyclic group of prime order. The lemma is true. Thus assume next that $H \cap N \neq 1$. Suppose $p$ is a prime and divides $|H \cap N|$, and $\tau \in H \cap N$ such that $o(\tau)=p$. Note that subgroups of a cyclic group are characeristic subgroups, so subgroups of $N$ are normal subgroups of $\operatorname{Aut}(G)$, thus $K \cap N=1$ as $K$ is core-free in $H$. In particular, $(H \cap N) \cap K=1$. Then $K<\langle\tau\rangle: K 6(H \cap N): K 6 H$. Since $K$ is maximal in $H$, we have $H \cap N=\langle\tau\rangle$ and $|H: K|=|\langle\tau\rangle|=p$ is a prime. This completes the proof of the lemma.

Now, we are ready to prove Theorem 1.1.

First, by Lemma $3.1, n=2$ and $G \cong D_{2 m}$ is a dihedral group.

By Lemma $2.2, X_{1} 6 \operatorname{Aut}(G, S) 6 \operatorname{Aut}(G)$ and $X_{1} \cong X_{1}^{S}$ is primitive, it then follows that $X_{1 s}$ is a core-free maximal subgroup of $X_{1}$, where $s \in S$. So, by Lemma 3.2, we 
conclude $\operatorname{val}(\Gamma)=|S|=\left|X_{1}: X_{1 s}\right|=p$ is a prime. Further, since $X_{1}$ is solvable, $X_{1}$ is an affine group, then there exist $\sigma, \tau \in \operatorname{Aut}(G, S)$ such that $X_{1}=\langle\sigma\rangle:\langle\tau\rangle \cong \mathbb{Z}_{p}: \mathbb{Z}_{r}$, where $r \mid(p-1)$ and $\langle\sigma\rangle$ is regular on $S$. That is, $S=s^{\langle\sigma\rangle}$. Since $\langle a\rangle$ is a characteristic subgroup of $G$ and has at most one involution, we conclude that $S \subseteq G \backslash\langle a\rangle$. Note that all involutions in $G \backslash\langle a\rangle$ are conjugate in $G$, without lose of generality, we may suppose $S=b^{\langle\sigma\rangle}$.

Suppose that $\sigma$ is defined by $\sigma: a \rightarrow a^{i}, b \rightarrow a^{j} b$, where $(i, m)=1$, and $06 j 6 m-1$. Then, by direct computation, we have $S=\left\{b, a^{j} b, \ldots, a^{\left(i^{p-2}+i^{p-3}+\cdots+1\right) j} b\right\}$. In particular, $\langle S\rangle=\left\langle b, a^{j}\right\rangle$. By the connectivity of $\Gamma,\left\langle b, a^{j}\right\rangle=G$, it then follows $(j, m)=1$. Further, as $o(\sigma)=p, b^{\sigma^{p}}=b$, we conclude $i^{p-1}+i^{p-2}+\cdots+1 \equiv 0(\bmod m)$. In particular, $i^{p} \equiv 1(\bmod m)$. Hence $i^{p-1}+i^{p-2}+\cdots+1 \equiv 0\left(\bmod p_{k}^{r_{k}}\right)$ for each $k$. If $i \equiv 1\left(\bmod p_{k}^{r_{k}}\right)$, then $p=p_{k}$ and $r_{k}=1$; if $i \not \equiv 1\left(\bmod p_{k}^{r_{k}}\right)$, then $p$ is the smallest positive solution of the equation $i^{x} \equiv 1\left(\bmod p_{k}^{r_{k}}\right)$. So $p \mid \varphi\left(p_{k}^{r_{k}}\right)$, where $\varphi$ is the Euler function. Thus we always have $p \mid p_{k}\left(p_{k}-1\right)$ for each $k$. Further, as $p_{1}<p_{2}<\cdots<p_{t}$, for $k \dot{z} 2, p_{k}$ not divides $p_{1}\left(p_{1}-1\right)$, so $p \neq p_{k}$, we thus conclude $p \mid\left(p_{1}\left(p_{1}-1\right), p_{2}-1, \ldots, p_{t}-1\right)$.

Suppose $m=p_{1}$ is a prime. Then $X=\hat{G}: X_{1}$, and $\mathbb{Z}_{p} 6 X_{1} 6 \mathbb{Z}_{p}: \mathbb{Z}_{p-1}$. Suppose next $m$ is not a prime. Since $i^{p-1}+i^{p-2}+\cdots+1 \equiv 0(\bmod m)$, it follows $i \not \equiv 1(\bmod m)$, and then $p$ is the smallest positive solution of the equation $i^{x} \equiv 1(\bmod m)$. So, if $\sigma^{\tau}=\sigma^{l}$, where $16 l 6 p-1$, then $a^{i}=a^{\sigma}=a^{\sigma^{\tau}}=a^{\sigma^{l}}=a^{i^{l}}$, so $i^{l-1} \equiv 1(\bmod m)$, we conclude $p \mid(l-1)$, and then $l=1$. Therefore, $X_{1} \cong X_{1}^{S} \cong \mathbb{Z}_{p}: \mathbb{Z}_{r}$ is abelian. Hence $r=1$ and $X_{1} \cong \mathbb{Z}_{p}$.

From the process of the above proof, we easily have the following corollary, which will be used later.

Corollary 3.3 Let $G=\langle a\rangle:\langle b\rangle \cong D_{2 m}$. Let $\sigma \in$ Aut $(G)$ such that $\sigma: a \rightarrow a^{i}, b \rightarrow a^{j} b$. Then $o(\sigma)=p$ and $\left\langle b^{\langle\sigma\rangle}\right\rangle=G$ if and only if $i^{p-1}+i^{p-2}+\cdots+1 \equiv 0(\bmod m)$ and $(j, m)=1$.

\section{Constructions}

Let $\Gamma=\operatorname{Cay}(G, S)$ be a connected $X$-locally primitive normal Cayley graph, where $G \cong$ $\mathbb{Z}_{m} \cdot \mathbb{Z}_{n}$ is a nonabelian metacyclic group, and $\hat{G}<X 6 \operatorname{Aut}(\Gamma)$. By Theorem 1.1, we know that $G \cong D_{2 m}$ is a dihedral group. In this section, we first construct three types of Cayley graphs of dihedral groups.

Construction 4.1 Suppose $m=p \dot{3} 3$ is a prime.

$$
\text { Let } G=\langle a\rangle:\langle b\rangle \cong D_{2 p} \text {. Let } S=G \backslash\langle a\rangle \text {, and } \Gamma=\operatorname{Cay}(G, S) \text {. }
$$

Construction 4.2 Suppose $m=p_{1}^{r_{1}} p_{2}^{r_{2}} \cdots p_{t}^{r_{t}}$ is not a prime, where $p_{1}<p_{2}<\cdots<p_{t}$, and $p$ is a prime such that $p \mid\left(p_{1}-1, p_{2}-1, \ldots, p_{t}-1\right)$.

Let $\left\langle a_{k}\right\rangle \cong \mathbb{Z}_{p_{k}^{r_{k}}}$. Take $\sigma_{k} \in \operatorname{Aut}\left(\left\langle a_{k}\right\rangle\right)$ of order $p$ for each $k$. Let $a=a_{1} a_{2} \cdots a_{t} \in$ $\left\langle a_{1}\right\rangle \times\left\langle a_{2}\right\rangle \times \cdots \times\left\langle a_{t}\right\rangle$, and $\tau=\sigma_{1} \sigma_{2} \cdots \sigma_{t} \in \operatorname{Aut}(\langle a\rangle)$. Let $G=\langle a\rangle:\langle b\rangle \cong D_{2 m}$. Define 
$\sigma \in \operatorname{Aut}(G)$ such that $\sigma: a \rightarrow a^{\tau}, b \rightarrow a^{j} b$, where $(j, m)=1$. Let $S=b^{\langle\sigma\rangle}$ and $\Gamma=$ $\operatorname{Cay}(G, S)$.

Construction 4.3 Suppose $m=p_{1}^{r_{1}} p_{2}^{r_{2}} \cdots p_{t}^{r_{t}}$ is not a prime, where $p_{1}<p_{2}<\cdots<p_{t}$, and $p_{1} \mid\left(p_{2}-1, \ldots, p_{t}-1\right)$, and suppose that the equation $x^{p_{1}-1}+x^{p_{1}-2}+\cdots+1 \equiv 0\left(\bmod p_{1}^{r_{1}}\right)$ has integer solution.

Let $\left\langle a_{k}\right\rangle \cong \mathbb{Z}_{p_{k}^{r_{k}}}$. Take $\sigma_{1} \in \operatorname{Aut}\left(\left\langle a_{1}\right\rangle\right)$, say $\sigma_{1}\left(a_{1}\right)=a_{1}^{m_{1}}$, such that $m_{1}^{p_{1}-1}+m_{1}^{p_{1}-2}+$ $\cdots+1 \equiv 0\left(\bmod p_{1}^{r_{1}}\right)$. For $k \dot{z} 2$, take $\sigma_{k} \in \operatorname{Aut}\left(\left\langle a_{k}\right\rangle\right)$ of order $p_{1}$. Let $a=a_{1} a_{2} \cdots a_{t} \in$ $\left\langle a_{1}\right\rangle \times\left\langle a_{2}\right\rangle \times \cdots \times\left\langle a_{t}\right\rangle$, and $\tau=\sigma_{1} \sigma_{2} \cdots \sigma_{t} \in \operatorname{Aut}(\langle a\rangle)$. Let $G=\langle a\rangle:\langle b\rangle \cong D_{2 m}$. Define $\sigma \in \operatorname{Aut}(G)$ such that $\sigma: a \rightarrow a^{\tau}, b \rightarrow a^{j} b$, where $(j, m)=1$. Let $S=b^{\langle\sigma\rangle}$ and $\Gamma=$ Cay $(G, S)$.

Lemma 4.4 Let $\langle c\rangle \cong \mathbb{Z}_{q}$ be a cyclic group, where $q=r^{s}$ is a prime power. Let $\sigma \in$ Aut $(\langle c\rangle)$ such that $\sigma: c \rightarrow c^{i}$. Suppose $o(\sigma)=p$ is a prime such that $(p, q)=1$. Then $i^{p-1}+i^{p-2}+\cdots+1 \equiv 0(\bmod q)$.

Proof. Since $o(\sigma)=p$, we have $i^{p} \equiv 1(\bmod q)$. So, to prove the lemma, it is sufficient to prove $(i-1, q)=1$. If this is not true, then $r \mid(i-1)$. Suppose $i=1+k r$. Then $c^{\sigma^{r^{s-1}}}=c^{(1+k r)^{r^{s-1}}}=c$. So $p \mid r^{s-1}$ as $o(\sigma)=p$, which is impossible.

The next lemma shows that graphs in Constructions 4.1-4.3 are locally primitive normal Cayley graphs, which then forms a part of the proof of Theorem 1.3.

Lemma 4.5 Graphs in Constructions 4.1-4.3 are locally primitive normal Cayley graphs of dihedral groups.

Proof. Suppose $\Gamma$ is a graph as in Construction 4.1. Since $S$ contains all involutions of $G$, so $\langle S\rangle=G$ and $\operatorname{Aut}(G, S)=\operatorname{Aut}(G) \cong \mathbb{Z}_{p}: \mathbb{Z}_{p-1}$. Suppose $\operatorname{Aut}(G, S)=\langle\sigma\rangle:\langle\tau\rangle$. Let $X_{1}=\langle\sigma\rangle:\left\langle\tau^{k}\right\rangle$ with $k \mid(p-1)$, and $X=\hat{G}: X_{1}$. Then as $|S|=p$ is a prime, $X_{1} 6$ Aut $(\Gamma)$ is primitive on $\Gamma(\mathbf{1})=S$, so $\Gamma$ is a connected $X$-locally primitive normal Cayley graph of $D_{2 p}$.

Suppose $\Gamma$ is a graph as in Construction 4.2. Let $X_{\mathbf{1}}=\langle\sigma\rangle$ and $X=\hat{G}: X_{\mathbf{1}}$. Suppose $a^{\sigma}=a^{i}$. Then $a_{k}^{\sigma_{k}}=a_{k}^{i}$. Since $\left(o\left(\sigma_{k}\right), p_{k}^{r_{k}}\right)=\left(p, p_{k}^{r_{k}}\right)=1$ for each $k$, by Lemma 4.4, $i^{p-1}+i^{p-2}+\cdots+1 \equiv 0\left(\bmod p_{k}^{r_{k}}\right)$, then $i^{p-1}+i^{p-2}+\cdots+1 \equiv 0(\bmod m)$. By Corollary 3.3 , $o(\sigma)=p$ and $\langle S\rangle=G$. As $|S|=p$ is a prime, $X_{\mathbf{1}}$ is primitive on $\Gamma(\mathbf{1})=S$, so $\Gamma$ is a connected $X$-locally primitive normal Cayley graph of $D_{2 m}$.

Finally, suppose $\Gamma$ is a graph as in Construction 4.3. Let $X_{1}=\langle\sigma\rangle$ and $X=\hat{G}$ : $X_{1}$. Suppose $a^{\sigma}=a^{i}$. For $k \dot{z} 2$, since $\left(o\left(\sigma_{k}\right), p_{k}^{r_{k}}\right)=1$, by Lemma $4.4, i^{p_{1}-1}+i^{p_{1}-2}+\cdots+1 \equiv$ $0\left(\bmod p_{k}^{r_{k}}\right)$. Moreover, by assumption, $m_{1}^{p_{1}-1}+m_{1}^{p_{1}-2}+\cdots+1 \equiv 0\left(\bmod p_{1}^{r_{1}}\right)$, we conclude $i^{p_{1}-1}+i^{p_{1}-2}+\cdots+1 \equiv 0(\bmod m)$. So, by Corollary $3.3, o(\sigma)=p$ and $\langle S\rangle=G$. Hence $\Gamma$ is a connected $X$-locally primitive normal Cayley graph of $D_{2 m}$ as $X_{1}$ is primitive on $\Gamma(\mathbf{1})=S$.

Now, we prove Theorem 1.3. 
By Lemma 4.5, to prove Theorem 1.3, it is sufficient to prove that every connected locally primitive normal Cayley graph of metacyclic group is isomorphic to a graph as in Constructions 4.1-4.3.

Let $\Gamma=$ Cay $(G, S)$ be a connected $X$-locally primitive Cayley graph of a metacyclic group. By Theorem 1.1, $G$ is a dihedral group. Suppose $G=\langle a\rangle:\langle b\rangle \cong D_{2 m}$ and $m=$ $p_{1}^{r_{1}} p_{2}^{r_{2}} \cdots p_{t}^{r_{t}}$, where $p_{1}<p_{2}<\cdots<p_{t}$. By Lemma 1.1, $\operatorname{val}(\Gamma)=p$ is a prime and $p \mid\left(p_{1}\left(p_{1}-1\right), p_{2}-1, \ldots, p_{t}-1\right)$.

Suppose first $p \nmid \backslash m$. Then $p \mid\left(p_{1}-1, p_{2}-1, \ldots, p_{t}-1\right)$. By Theorem 1.1, we may suppose $X_{1}=\langle\sigma\rangle \cong \mathbb{Z}_{p}$, and $S=b^{\langle\sigma\rangle}$, where $\sigma \in$ Aut $(G)$. Suppose $\sigma: a \rightarrow a^{i}, b \rightarrow a^{j} b$. By Corollary 3.3, $i^{p-1}+i^{p-2}+\cdots+1 \equiv 0(\bmod m)$ and $(j, m)=1$. Consider $\sigma$ as an automorphism of the cyclic group $\langle a\rangle$. It is easily shown that there exist elements $a_{1}, a_{2}, \ldots, a_{t} \in\langle a\rangle$ such that $o\left(a_{k}\right)=p_{k}^{r_{k}}$ and $a=a_{1} a_{2} \cdots a_{t}$. Let $m_{k} \equiv i\left(\bmod p_{k}^{r_{k}}\right)$. Define $\sigma_{k}: a_{k} \rightarrow a_{k}^{m_{k}}$. Then $\sigma=\sigma_{1} \sigma_{2} \cdots \sigma_{t}$. Moreover, as $m_{k}^{p-1}+m_{k}^{p-2}+\cdots+1 \equiv 0\left(\bmod p_{k}^{r_{k}}\right)$, and $p \neq p_{k}$, we have $m_{k}^{p} \equiv 1\left(\bmod p_{k}^{r_{k}}\right)$ and $m_{k} \neq 1\left(\bmod p_{k}^{r_{k}}\right)$, so $\sigma_{k}$ is an automorphism of $\left\langle a_{k}\right\rangle$ of order $p$. Hence $\Gamma$ is isomorphic to a graph as in Construction 4.2.

Suppose now $p \mid m$. Then $p=p_{1}$ is a prime. If $m=p_{1}$, then $S=D_{2 p} \backslash\langle a\rangle$, and $\Gamma=\operatorname{Cay}\left(D_{2 p}, S\right)$ is isomorphic to a graph as in Construction 4.1. If $m$ is not a prime, then $t ; 2$ and $p_{1} \mid\left(p_{2}-1, \ldots, p_{t}-1\right)$. By Theorem 1.1 , we may suppose $X_{1}=\langle\sigma\rangle \cong \mathbb{Z}_{p_{1}}$, and $S=b^{\langle\sigma\rangle}$, where $\sigma \in \operatorname{Aut}(G)$. Suppose $\sigma: a \rightarrow a^{i}, b \rightarrow a^{j} b$. By Corollary 3.3, $i^{p_{1}-1}+i^{p_{1}-2}+\cdots+1 \equiv 0(\bmod m)$ and $(j, m)=1$. View $\sigma$ as an automorphism of the cyclic group $\langle a\rangle$, then with similar argument as in the above paragraph, one may prove: there exist elements $a_{1}, a_{2} \ldots, a_{t} \in\langle a\rangle$ such that $o\left(a_{k}\right)=p_{k}^{r_{k}}$ and $a=a_{1} a_{2} \cdots a_{t}$; there exist $\sigma_{k} \in \operatorname{Aut}\left(\left\langle a_{k}\right\rangle\right)$ such that $\sigma=\sigma_{1} \sigma_{2} \cdots \sigma_{t}$; for $k \dot{z} 2, \sigma_{k}$ are of order $p_{1}$; if suppose

$\sigma_{1}\left(a_{1}\right)=a_{1}^{m_{1}}$, then $m_{1}^{p_{1}-1}+m_{1}^{p_{1}-2}+\cdots+1 \equiv 0\left(\bmod p_{1}^{r_{1}}\right)$. So $\Gamma$ is isomorphic to a graph as in Construction 4.3.

\section{Locally primitive bi-normal dihedral graphs}

Let $\Gamma=\operatorname{Cay}(G, S)$, and $\hat{G}<X 6$ Aut $(\Gamma)$. If $\hat{G}$ is not normal in $X$, but has a subgroup of index 2 which is normal in $X$, then $\Gamma$ is called an $X$-bi-normal Cayley graph of $G$. For a group $H$, recall the socle of $H$, denoted by $\operatorname{soc}(H)$, is the products of all minimal subgroups of $H$. In this final section, we give a characterization of locally primitive bi-normal Cayley graphs of dihedral groups.

Theorem 5.1 Let $\Gamma=\operatorname{Cay}(G, S)$ be a connected $X$-locally primitive bi-normal Cayley graph of valency at least 3 , where $G \cong D_{2 m}$ is a dihedral group, and $\hat{G}<X 6$ Aut $(\Gamma)$. Then $\Gamma \cong \mathrm{K}_{m, m}$ is a complete bi-partite graph, or val $(\Gamma)=4$ or $p$ with $p$ a prime.

Proof. By assumption, there exists a subgroup $M$ of $\hat{G}$ with index 2 such that $M$ is normal in $X$. Then $M \cong \mathbb{Z}_{m}$ or $D_{m}$ and $m$ is even. Let $\Delta_{1}, \Delta_{2}$ be the $M$-orbits on $V(\Gamma)$. Let $X^{+}=X_{\Delta_{1}}=X_{\Delta_{2}}$.

Assume first $X^{+}$is unfaithful on $\Delta_{1}$. Let $K_{1}$ be the kernel of $X^{+}$acting on $\Delta_{1}$. Then $K_{1} \neq 1$ and $K_{1}$ acts faithfully on $\Delta_{2}$. For an edge $\{\alpha, \beta\}$ of $\Gamma$, where $\alpha \in \Delta_{1}$ and $\beta \in \Delta_{2}$, 
let $B$ be the $K_{1}$-orbit of $\beta$ in $\Delta_{2}$. Since $K_{1}$ fixes $\alpha$, we conclude $B \subseteq \Gamma(\alpha)$. Further, as $1 \neq K_{1}^{\Gamma(\alpha)} \triangleleft\left(X_{\alpha}^{+}\right)^{\Gamma(\alpha)}=X_{\alpha}^{\Gamma(\alpha)}$, and $X_{\alpha}^{\Gamma(\alpha)}$ is primitive, we have $B=\Gamma(\alpha)$. Since this holds for every vertex which is adjacent to a vertex of $B$, by the connectivity of $\Gamma$, we have $B=\Delta_{2}$, that is, $\Gamma \cong \mathrm{K}_{m, m}$. Similarly, the same result holds if $X^{+}$is unfaithful on $\Delta_{2}$.

Thus assume next $X^{+}$is faithful on $\Delta_{1}$ and $\Delta_{2}$. Since $M \triangleleft X^{+}$and $M$ is regular on $\Delta_{1}$, it follows $X^{+} 6 \mathbf{N}_{\text {Sym }\left(\Delta_{1}\right)}(M) \cong M$ :Aut $(M)$, see [4, Corollary 4.2B]. Then $X_{\alpha}=X_{\alpha}^{+} 6$ Aut $(M)$, where $\alpha \in V(\Gamma)$.

Suppose $M \cong \mathbb{Z}_{m}$. Then $X_{\alpha} 6$ Aut $\left(\mathbb{Z}_{m}\right)$ is abelian. Since $\Gamma$ is $X$-locally primitive, $X_{\alpha}^{\Gamma(\alpha)}$ is an abelian primitive permutation group, so $\operatorname{val}(\Gamma)=|\Gamma(\alpha)|$ is a prime.

Suppose now $M \cong D_{m}$ and $m$ is even. Then $X_{\alpha} 6$ Aut $(M)$ is soluble. Further, as $X_{\alpha}^{\Gamma(\alpha)}$ is primitive, it follows that $X_{\alpha}^{\Gamma(\alpha)}$ is affine, $\operatorname{sos} \operatorname{soc}\left(X_{\alpha}^{\Gamma(\alpha)}\right)=\mathbb{Z}_{p}^{d}$, and $\operatorname{val}(\Gamma)=|\Gamma(\alpha)|=p^{d}$ for some prime $p$. Let $C=\mathbf{C}_{X}(M)$ be the centralizer of $M$ in $X$, and $D=\langle C, M\rangle$. Then $C, D$ are normal subgroups of $X$, and $C^{+}:=C_{\Delta_{1}}=\mathbf{C}_{X^{+}}(M), D^{+}:=D_{\Delta_{1}}=\left\langle C^{+}, M\right\rangle$ are normal subgroups of $X^{+}$. Let $g \in G \backslash M$. In what follows, we distinguish two cases, depending on whether $g \in \mathbf{C}_{X}(M)$ or not.

Case 1. $g \in \mathbf{C}_{X}(M)$.

In this case, $D^{+}=\left\langle D^{+}, g\right\rangle \cong D^{+} \mathbb{Z}_{2}$ is transitive on $V(\Gamma)$. If $D_{\alpha}=1$, then $G=D$ is a normal subgroup of $X$, that is, $\Gamma$ is an $X$-normal Cayley graph of $G$, which is not the case. Thus $D_{\alpha} \neq 1$. Then $D_{\alpha}^{\Gamma(\alpha)} \neq 1$, and $\operatorname{soc}\left(X_{\alpha}^{\Gamma(\alpha)}\right)=\mathbb{Z}_{p}^{d} \triangleleft D_{\alpha}^{\Gamma(\alpha)}$. Moreover, $D_{\alpha}=D_{\alpha}^{+} \cong M D_{\alpha}^{+} / M \cong D^{+} / M \cong \mathbf{C}^{+} / Z(M)$ is a factor group of $C^{+}$. Further, as $M$ is regular on $\Delta_{1}, C^{+} 6 M \cong D_{m}$ is semiregular on $\Delta_{1}$. Note that $D_{\alpha}^{\Gamma(\alpha)}$ is a factor group of $C^{+} / Z(M)$, and subgroups and factor groups of a dihedral group are dihedral or cyclic, it follows that $\mathbb{Z}_{p}^{d}$ is a subgroup of a dihedral group or a cyclic group, we then conclude that either $d=1$ or $d=2$ and $p=2$. That is, $\operatorname{val}(\Gamma)=p$ or 4 .

Case 2. $g \notin \mathbf{C}_{X}(M)$.

In this case, $C=C^{+}$and $D=D^{+}$, so $D$ has 2 orbits on $V(\Gamma)$. If $C=1$, then $D=M$, and $X / M 6 \operatorname{Aut}(M) / M \cong \operatorname{Aut}\left(\mathbb{Z}_{m / 2}\right) / \mathbb{Z}_{2}$ is abelian. Further, as $X^{+}=M: X_{\alpha}^{+}$, we have $X_{\alpha}^{+} \cong X^{+} / M 6 X / M$ is abelian, so $X_{\alpha}^{\Gamma(\alpha)}$ is an abelian primitive group, thus $\operatorname{val}(\Gamma)=$ $|\Gamma(\alpha)|$ is a prime. If $C \neq 1$, then $D_{\alpha} \neq 1$, so $D_{\alpha}^{\Gamma(\alpha)} \neq 1$ and $\operatorname{soc}\left(X_{\alpha}^{\Gamma(\alpha)}\right)=\mathbb{Z}_{p}^{d} \triangleleft D_{\alpha}^{\Gamma(\alpha)}$. Now, with similar argument as in Case 1 , one may prove that $\operatorname{val}(\Gamma)=p$ or 4 . This completes the proof of the theorem.

\section{Acknowledgment}

The author would like to thank the School of Mathematics and Statistics, The University of Western Australia for its hospitality. The first draft of this paper was done when the author visited there. The author also thanks the anonymous referee for the helpful comments and suggestions. 


\section{References}

[1] B. Alspach, M. Conder, D. Marušič and M. Y. Xu, A classification of 2-arc-transitive circulants, J. Algebraic Combin. 5 (1996), 83-86.

[2] B. G. Basmaji, On the isomorphisms of two metacyclic groups, Proc. Amer. Math. Soc. 22 (1969), 175-182.

[3] N. Biggs, Algebraic Graph Theory, Cambridge University Press, 2th Ed, 1992, New York.

[4] J. D. Dixon, B. Mortimer, Permutation Groups, Springer, 1996.

[5] C. D. Godsil, On the full automorphism group of a graph, Combinatorial, 1 (1981), 243-256.

[6] A. A. Ivanov and C. E. Praeger, On finite affine 2-arc transitive graphs, Europ. J. Combin. 14 (1993), 421-444.

[7] C. H. Li, C. E. Praeger, A. Venkatesh and S. Zhou, Finite locally-primitive graphs, Discrete Math. 246 (2002), 197-218.

[8] C. H. Li, Finite edge-transitive Cayley graphs and rotary Cayley maps, Trans. Amer. Math. Soc. 358 (2006), 4605-4635.

[9] C. H. Li, J. Pan, Finite 2-arc-transitive abelian Cayley graphs, Europ. J. Combin. 29(2007), 148-158.

[10] C. H. Li, J. Pan, Locally primitive graphs of prime-power order, J. Aust. Math. Soc. 86 (2009), 111-122.

[11] D. Marušič, On 2-arc-transitivity of Cayley graphs, J. Combin. Theory Ser. B, 87 (2003), 162-196.

[12] C. E. Praeger, On O'Nan-Scott theorem for finite quasiprimitive permutation groups and an application to 2-arc transitive graphs, J. London. Math. Soc. 47 (1992), 227239.

[13] C. E. Praeger, Finite normal edge-transitive Cayley graphs, Bull. Aust. Math. Soc. 60 (1999), 207-220.

[14] D. J. S. Robinson, A Course in the Theory of Groups, Springer-Verlag, New York, 1982.

[15] M. Y. Xu, Automorphism groups and isomorphisns of Cayley digraphs, Discrete Math. 182 (1998), 309-320.

[16] M. Y. Xu, Introduction of Finite Groups, Chinese Science Press, 1999. 